\title{
Response of patients with postencephalitic Parkinsonism to levodopa ${ }^{1}$
}

\author{
ROGER C. DUVOISIN, JOAO LOBO-ANTUNES, AND \\ MELVIN D. YAHR \\ From the Clinical Center for Research in Parkinsonism, College of Physicians and Surgeons, \\ Columbia University, and The Neurological Institute, Columbia-Presbyterian Medical Center, \\ New York, U.S.A.
}

SUMmaRY Thirty patients with postencephalitic Parkinsonism were treated with levodopa. In seven patients, treatment was withdrawn because of adverse reactions or insufficient benefit. Improvements observed in 26 patients who remained on treatment for a sufficient period to permit adequate assessment were comparable with those previously reported in Parkinson's disease. Some sequelae of encephalitis lethargica-notably, oculogyria and drooling-were appreciably improved in most patients affected, whereas choreoathetoid dyskinesias were aggravated by levodopa. Adventitious choreiform involuntary movements and behaviour disorders were the major side-effects. Patients with postencephalitic Parkinsonism may respond as well as patients with Parkinson's disease to levodopa but they develop both favourable responses and side-effects at a lower dosage and require careful dosage adjustments and close surveillance.

There is general agreement that levodopa is the most effective therapeutic agent presently available for the treatment of Parkinsonism. The general principles underlying its use in clinical practice, its effectiveness, its side-effects and its limitations are now well documented in an extensive and rapidly growing literature. However, nearly all the data reported to date concern the use of levodopa in patients with Parkinson's disease. To our knowledge, only two reports (Calne, Stern, Lawrence, Sharkey, and Armitage, 1969; Krasner and Cornelius, 1970)_plus several 'letters to the editor' (Sacks and Kohl, 1970a, b; Sacks, Kohl, Schwartz, and Messelhoff, 1970c; Sacks, Messelhoff, and Schwartz, 1970d) -dealing specifically with the use of levodopa in postencephalitic Parkinsonism have been published. It is difficult to draw any firm conclusions from the small number of patients described, and the relatively short periods over which they were observed, regarding the indications, contraindications, and efficacy of

\footnotetext{
1 Supported in part by The National.Institutes of Health, NINDS under Grant Number NB 05 184, and the Parkinson's Disease Foundation, New York, New York.
}

levodopa in this distinctive and specific aetiological type of Parkinsonism. Moreover, little has been noted about the effects of levodopa on important sequelae of encephalitis lethargica other than Parkinsonism such as oculogyric crises, ocular and bulbar palsies, tics, dystonic postures and movements, or the sleep and behavioural disorders commonly encountered in postencephalitic patients. Accordingly, we think it worthwhile to report our experience with long-term levodopa therapy in a large series of patients with a well-documented diagnosis of postencephalitic Parkinsonism.

\section{METHODS}

Thirty-three patients considered to have postencephalitic Parkinsonism were included among a large number of patients admitted to a clinical investigation of levodopa in the treatment of Parkinsonism conducted at the Columbia-Presbyterian Medical Center. All were personally interviewed and examined. After a careful review of all the available clinical data, they were classified as 'definitely', 'probably', or 'possibly' postencephalitic according to criteria defined in a previous study 
of postencephalitic Parkinsonism (Duvoisin and Yahr, 1965). (Sixteen of the patients had been among the subjects of that study.) Two patients whose clinical picture was complicated by brain tumour and one with a slowly progressive atypical Parkinsonism syndrome who was considered to be only 'possibly' postencephalitic were omitted from the present analysis.

There remained 30 patients who were considered to be either definitely or probably postencephalitic (Table 1). Twenty-six were classified as 'definitely' postencephalitic. Twenty-three of these had both a

TABLE 1

DIAGNOSTIC DETAILS

\begin{tabular}{llc}
\hline $\begin{array}{l}\text { Certainty of } \\
\text { diagnosis }\end{array}$ & \multicolumn{1}{c}{ Criteria employed for diagnosis } & $\begin{array}{c}\text { No. of } \\
\text { patients }\end{array}$ \\
\hline Definite & $\begin{array}{l}\text { a. Documented history of encephalitis } \\
\text { lethargica and characteristic sequelae } \\
\text { b. Oculogyria but no history of } \\
\text { encephalitis lethargica }\end{array}$ & 23 \\
c. Characteristic post-mortem features & 1 \\
Probable & $\begin{array}{l}\text { Characteristic non-Parkinsonian sequelae } \\
\text { of encephalitis lethargica }\end{array}$ & 4 \\
\hline & \multicolumn{2}{c}{ Total } \\
\hline
\end{tabular}

reliable or documented history of encephalitis lethargica and characteristic sequelae; two had no definite history of encephalitis but had oculogyric crises. One patient had a vague history of encephalitis circa 1920, and had been classified as 'possibly' postencephalitic in the earlier study cited above (see case 3 ). This patient has since died after a period of levodopa therapy. Characteristic features of postencephalitic Parkinsonism were found on postmortem study, and we have consequently reclassified the case as 'definitely' postencephalitic (case 1, below).

Four patients were classified as 'probably' postencephalitic. They had neither a satisfactory history of encephalitis nor oculogyric crises, but otherwise presented the typical clinical features of postencephalitic Parkinsonism including neurological abnormalities frequently seen in postencephalitics.

The patients ranged in age from 55 to 80 years of age (mean 60.4 years) and included 18 men and 12 women. The age of onset ranged from 12 to 50 years (mean 29.4 years). The duration of illness at the time levodopa therapy was initiated ranged from 16 to 49 years (mean 31.7 years).

Three patients had involuntary choreiform movements before commencing levodopa therapy. Two had an orofacial dyskinesia characterized by pro trusion of the tongue, opening of the mouth, and lateral deviation of the jaw and one had choreo $Z$ athetotic movements of the left upper limb. Nine patients had oculogyric crises.

The severity of the Parkinsonism was assessed weekly or more frequently during the initial periods 0 of treatment and at each follow-up visit. The degree of disability was evaluated according to a five stage $\frac{?}{2}$. scale previously described by Hoehn and Yahr? (1967) defined as follows: stage I, early unilateraf. signs and symptoms; stage II, bilateral signs and symptoms mild in degree; stage III, the complete syndrome with beginning impairment of equilibrium $: \frac{C}{0}$ stage IV, severe Parkinsonism with significant dis $\overline{\bar{n}}$ ability but the patient can walk and attend to personal needs with assistance; stage $\mathrm{V}$, complete invalidism with the patient confined to bed andes chair.

Rigidity, tremor, speech, facial expression, seborrhoea, salivation, bradykinesia, the manner of rising from a chair, posture, gait, postural stability finger dexterity, and the performance of rapid alternating movements with hands and feet werew evaluated in a standard manner and each item was rated on a carefully defined five point scale. The sump of the ratings constituted a score which was con sidered an index of the overall severity of Parkinsof -0 ism. The difference between treatment and pR-treatment scores was divided by the latter and the result multiplied by 100 to calculate the percentage of improvement in each case. Special attention was directed to the incidence and duration of the ocu通- $\overrightarrow{0}$ gyric crises.

Complete physical examination and screening tests were carried out in all patients in order too exclude cardiovascular, renal, hepatic, or haemato-logical disorders. Blood counts, urinalyses, andō routine blood chemical analyses were performed ato each visit in all patients. Electrocardiograms were performed before commencing levodopa and re- $\overrightarrow{\vec{o}}$ peated at varying intervals.

Our method of treatment was to continue each patient's previous medication and add levodopa in? small initial doses, using tablets or capsules of $250 \mathrm{mg}$ and $500 \mathrm{mg}$. The dosage and schedule were modified according to the responses observed in each:patient with a high degree of individualization in order to achieve the best compromise betweenô benefits and side effects. The average daily dose of $₹$ levodopa was $2.8 \mathrm{~g}$. An effort was made to dis-o continue previous anti-Parkinsonism drugs after $a_{\triangle}$ response to levodopa was observed but results were을 nearly always better with a combination of two or more types of drugs and only two patients remained.s on levodopa alone. Eleven patients received two or 0 
three anti-Parkinsonism agents, mainly of the anticholinergic type, in addition to levodopa. Amantadine $\mathrm{HCl}$ was also employed in four patients.

\section{RESULTS}

Twenty-two of the thirty patients were still on levodopa therapy at the time of writing. One patient died during the 10th month of treatment. Levodopa was discontinued in seven patients for reasons to be detailed below after periods of time ranging from one to 11 months. Four were discontinued after less than three months of treatment. Three were discontinued after longer periods; one after four months, another after eight months, and the third after 11 months. We feel that these three patients were treated for a sufficient period of time to permit a fair assessment of the benefits as well as the side-effects of levodopa therapy. Accordingly, we calculated the therapeutic improvements obtained in a total of 26 patients who remained on levodopa therapy for a minimum of four to a maximum of 38 months (mean 16.8 months). The results are shown in Table 2 .

TABLE 2

RESULTS OF TREATMENT WITH LEVODOPA

\begin{tabular}{lccccccc}
\hline $\begin{array}{l}\text { Oterall improvement } \\
(\%)\end{array}$ & \multicolumn{5}{c}{ Pretrial clinical stage } & $\begin{array}{c}\text { No. of } \\
\text { patients }\end{array}$ \\
\cline { 2 - 7 } & $I$ & $I I$ & III & IV & $V$ & \\
\hline $80-100$ & - & 2 & - & - & 1 & 3 \\
$50-79$ & 1 & 3 & $5 \dagger$ & 2 & 1 & 12 \\
$20-49$ & 1 & 2 & 3 & - & $1 \mp$ & 7 \\
$0-19$ & 2 & $1^{*}$ & 1 & - & - & 4 \\
\hline Total & 4 & 8 & 9 & 2 & 3 & 26 \\
\hline
\end{tabular}

* Discontinued after eight months because of malaise and vertigo. † One patient discontinued after four months because of adventitious involuntary movements.

$\ddagger$ Discontinued after 11 months because of cardiac arrhythmia.

Fifteen of the 26 patients obtained a reduction in their examination scores exceeding $50 \%$; in one the stigmata of Parkinsonism virtually disappeared. In seven of the remaining 11 patients, improvements were limited but significant allowing more independence in the performance of daily living activities. Both the major and minor features of the disease seemed to respond. Hypersomnolence was greatly reduced or abolished. Reduction of drooling and improvement of long-lasting postural abnormalities were observed, but neither ocular palsies nor the paresis of ocular convergence so characteristic of postencephalitics were altered.

Disability was reduced in 16 patients. Three initially classified as stage $\mathrm{V}$ were markedly improved, two to stage II and one to stage III. Two patients whose disability was graded as stage IV also enjoyed significant reduction of disability, one to stage III and the other to stage II.

Oculogyric crises were present in nine patients. The approximate incidence of oculogyric crises before and after treatment is detailed in Table 3 .

TABLE 3

INCIDENCE OF OCULOGYRIC CRISES

\begin{tabular}{ccc}
\hline $\begin{array}{l}\text { Duration of treatment } \\
\text { (months) }\end{array}$ & \multicolumn{2}{c}{ Frequency of oculogyric crises per month } \\
\cline { 2 - 3 } & Before treatment & After treatment \\
\hline 14 & 4 & None \\
17 & 4 & 1 \\
10 & $8-12$ & 1 \\
7 & 1 & 1 \\
28 & $12-20$ & $12-20$ \\
21 & 4 & $12-20$ \\
38 & $12-16$ & $4-8$ \\
32 & 4 & 1 \\
7 & $8-12$ & 4 \\
\hline
\end{tabular}

The frequency and duration of the crises were significantly reduced in six patients. In two patients no change was noticed and in one a marked aggravation occurred. However, in this case the improvement of the other symptoms of Parkinsonism was remarkable (from stage $\mathrm{V}$ to stage II) and the patient felt that increased oculogyria was an acceptable price to pay for the improvement obtained. Two patients claimed that the ingestion of an additional dose of levodopa $(0.5$ to $1.5 \mathrm{~g})$ could stop a crisis within 30 minutes. In one patient, crises had occurred almost daily before levodopa, lasting several hours and sometimes subsiding only with sleep. Poor control was achieved with 'classical' medications, and the patient was compelled to retire from his occupation. Levodopa ( $2 \mathrm{~g}$ per day) added to the previous regimen (benzhexol (Artane) $35 \mathrm{mg}$ daily) reduced the incidence of 
the crises to one to two per week, and markedly reduced their severity and duration. On several occasions, the dosage of levodopa was reduced below $1.5 \mathrm{~g}$ daily; each time there was a return to the previous frequency and severity of oculogyric crises. The pattern has remained consistent over a follow-up period of 38 months.

One patient had exhibited for many years a curious and frequent 'grunting' which was socially very embarrassing to him and had not been controlled by any previous treatment. The grunting phenomenon or 'tic' completely disappeared with levodopa. In six patients an initially gratifying response to levodopa was lost when the subsequent advent of involuntary movements and behavioural disorders compelled a reduction of the dosage. Nevertheless, with careful titration of the dosage a compromise between benefit and side-effects was attained which still gave these patients a significant if less than ideal therapeutic response.

As noted above, levodopa therapy was ultimately discontinued in seven patients. In one patient it was withdrawn because of a cardiac arrhythmia which was considered to be lifethreatening. In the remainder it was withdrawn because the side-effects (involuntary movements in two, malaise and vertigo in another, and behavioural disturbances in three) persisted to an unacceptable degree, despite reduction in the dosage to levels yielding little or no therapeutic benefit.

The patient who died during the 10th month of treatment apparently expired in his sleep. He was found dead in bed. No necropsy was performed and the cause of death is unknown. However, because of a previous history of myocardial infarction, a cardiac death may reasonably be suspected.

The side-reactions encountered are summarized in Table 4. Nausea and vomiting were reported by nine patients but did not represent a major difficulty, subsiding spontaneously in time or with adequate adjustment of the medication. Irregularity of the cardiac rhythm was noticed in three patients. Involuntary movements occurred in 19 patients, involving chiefly the facial musculature. In three patients, the movements consisted of bizarre respiratory patterns with irregular hyperpnoea. One patient complained of 'grunting'.
TABLE 4

INCIDENCE OF SIDE-EFFECTS

\begin{tabular}{lr}
\hline Side-effects & No. \\
\hline Nausea and vomiting & 9 \\
Cardiac arrhythmia & 3 \\
Involuntary movements & 19 \\
Psychic manifestations & 10 \\
Postural hypotension & 1 \\
Elevated serum nitrogen & 1 \\
\hline
\end{tabular}

The three patients who had choreiform involuntary movements before beginning levo- $\frac{\bar{c}}{\frac{1}{\sigma}}$ dopa treatment experienced an increase in these $\mathbb{Q}$ movements even on very small doses of levo- ڤ dopa. In one patient with a previous orofacial $\vec{\circ}$ dyskinesia the movements were intolerably severe even at doses of levodopa yielding relatively little improvement and precluded increasing the dosage to an effective level. This patient was consequently unable to benefit from levo- is dopa. However, the other two patients with previous dyskinetic phenomena obtained signifis cant benefit from doses of levodopa which di not increase their dyskinesia to unacceptab proportions. Postural hypotension was observed in only one patient and was mild and transiert in duration.

The psychic side-effects encountered warra a more detailed account. They occurred in nine patients but in only three was levodopa discontinued. These three patients became actively psychotic, with compulsive behaviour, confusion, and severe agitation. One had received levodopa with $\alpha$-methyldopa hydrazine (MK-486-a dopadecarboxylase inhibitor) and, although a dra- $\stackrel{2}{\not}$ matic improvement of the neurological status with a $95 \%$ reduction of the pretrial score was obtained in four days, a severe behavioural dis-? order rendered the patient unmanageable. The same symptoms recurred on treatment with levodopa alone at a very modest dose (1.5 g daily).

Six patients with behavioural disturbances were able to continue on levodopa treatment at a lower dosage and still derive significant $\frac{}{3}$ benefit. In one of these patients bizarre, socially $\frac{D}{0}$ inappropriate, behaviour which had occurred many years before seemed to be reactivated. $\mathrm{He} N$ would enter neighbouring homes without knock- N 
ing and uninvited, enter automobiles stopped temporarily at traffic lights and accost passers-by on the street to offer them a haircut (he had once been a barber). After a period of discontinuance the medication was resumed in a lower amount without recurrence of this curious behaviour. In another patient (case 2, below) a compulsive syndrome was observed, but the family felt that the improvements obtained rendering the patient essentially self-sufficient in his daily living activities justified continuing treatment. Two patients became very hyperactive when the dose of levodopa was increased above a certain level, but a satisfactory benefit was achieved with tolerable doses. A paranoid psychosis was induced in one patient on the 14th day of treatment with a dose of $4 \mathrm{~g}$ daily but was not evident on a subsequent dosage of $2 \mathrm{~g}$ daily. The patient continued to improve at this reduced dosage. Reduction of the levodopa to $4 \mathrm{~g}$ daily resulted in marked improvement in the mental status in another patient in whom $5 \mathrm{~g}$ daily induced confusion.

A reactive depression in a patient who made an unsuccessful suicide attempt was felt to be precipitated by disappointment with the incomplete reversal of Parkinsonian symptoms yielded by levodopa. No change in treatment was required.

Laboratory studies remained within normal limits except for one patient who had a transient elevation of the serum urea nitrogen.

Two patients suffered hip fractures during the period of observation on levodopa.

\section{CASE 1}

D.C., a 72 year old woman at the time of her death, had suffered from Parkinsonism most of her life. Her illness may be traced to an ill-defined episode of excessive sleepiness at the age of 22 years in 1920 . Ten years later she began to complain of general weakness and tremor of the left hand. In 1931 she consulted Dr. Douglas McAlpine in London who wrote that 'she presented a typical picture of Parkinsonism, the limbs on the right side being scarcely affected'. She had a severe personality disturbance; she was moody, and would sit around for many hours each day, not taking care of her home or children properly. Subsequently her emotional status gradually improved. When first seen at the New York Neurological Institute in 1932 she had a coarse tremor of the left hand and loss of associated movements of the left upper extremity when walking. The neurological examination was otherwise unremarkable. Two years later tremor of the right hand was noted. Subsequent progression of her illness was very gradual. In 1956 she began to develop a thoracic kyphosis. Her gait subsequently deteriorated and her voice became soft and increasingly difficult to understand. By 1957 she walked with short steps with loss of associated movements and had moderate rigidity and cogwheeling in all extremities and a coarse tremor of the hands. Rapid alternating and succession movements were performed poorly. Ocular convergence was impaired, and a hyperactive glabellar reflex was present. Trihexyphenidyl, $8 \mathrm{mg}$ daily, apparently gave some symptomatic improvement but by 1959 her equilibrium was severely impaired and she suffered frequent falls. Two years later she had occasional episodes of angina pectoris. Signs of congestive heart failure were noted.

She fell with increasing frequency, suffering a Colles's fracture of the right wrist, and several rib fractures and scalp lacerations. In 1965 she began to require increasing help in her activities of daily living and three years later had to be placed in a nursing home.

In November 1968 she was started on a trial of levodopa treatment. At that time the vital signs were normal. There was a soft precordial systolic murmur and mild ankle oedema. A severe dorsal kyphoscoliosis was present with rotation of the head to the right, the chin pressed against the right shoulder. There was a severe rigidity of the paraspinal muscles and extremities, more marked on the left. A minimal tremor of the fingers and tongue was present. The gait was short stepped, with severe festination, and she tended to fall spontaneously. Arising from a chair was difficult and sometimes impossible without assistance. There was a moderate facial masking. The voice was very soft and the speech barely intelligible. Pretrial investigations were normal except for the presence in the electroencephalogram (EEG) of intermittent bitemporal medium to high voltage slow and sharp waves and anterolateral depression of $\mathrm{T}$ waves in the electrocardiogram (ECG). Psychomotor testing revealed minimal memory loss and severe deficit on perceptual motor tasks. Previous medications were continued (trihexyphenidyl, $4 \mathrm{mg}$; diphenhydramine, $75 \mathrm{mg}$; and benztropine, $2 \mathrm{mg}$ per day) and levodopa was started at a daily dosage of $0.75 \mathrm{~g}$ and progressively increased. On $5 \mathrm{~g}$ daily she began to have mild nocturnal confusion. Trihexyphenidyl and benztropine were discontinued with improvement of the mental symptoms. However, two days later she developed a supraventricular arrhythmia and levo- 
dopa was withheld for several days until the ECG reverted to its pre-trial status. However, her signs of Parkinsonism which had shown significant improvement, again became markedly exacerbated. Levodopa was restarted in small doses and with gradual increments a daily total of $2.5 \mathrm{~g}$ was attained. A significant functional improvement was achieved, and this increased slightly in the following months. A slight improvement of the severe kyphotic deformity was noticed and she was able to dress herself independently. In September 1969 frequent episodes of palpitations, accompanied by pain in the chest and dyspnoea, occurred. Levodopa was again discontinued.

The patient subsequently died of aspiration pneumonia in February 1970. A necropsy was performed and histological and biochemical studies were carried out on the brain. On gross examination of the brain there was depigmentation of the zona compacta of the substantia nigra. Pathological changes on microscopic study included: granulovacuolar degeneration and neurofibrillary tangles in nerve cells of the pyramidal layer of the hippocampus, marked arteriosclerotic changes in the walls of the small arteries in the caudate nucleus and putamen, a small cystic area of encephalomalacia in the right putamen; and neurofibrillary tangles in the locus caeruleus, the substantia innominata, and the paraventricular nuclei of the hypothalamus. There was a moderate loss of neuromelanin-pigmented neurones in the zona compacta of the substantia nigra and locus caeruleus with extracellular deposits of pigment. There was also neuronal loss in the dorsal vagus nucleus. No Lewy bodies were found.

Assays of various subcortical structures done by Dr. Lucien Cote revealed very low levels of dopamine: $0.03 \mu \mathrm{g} / \mathrm{g}$ in the caudate nucleus and putamen and $0.02 \mu \mathrm{g} / \mathrm{g}$ in the pallidum. The mean normal control values for these structures were $3 \cdot 90,4 \cdot 10$, and $0.50 \mu \mathrm{g} / \mathrm{g}$ respectively; the mean values from eight patients with Parkinson's disease were $1 \cdot 21$, $0 \cdot 40$, and $0 \cdot 18 \mu \mathrm{g} / \mathrm{g}$ in the corresponding areas (Cote, Yahr, Duvoisin, Wolf, and Marksbery, 1970).

COMMENT From the earliest work with levodopa, the risk of provoking cardiac arrhythmias in elderly individuals with arteriosclerotic cardiovascular disease has been a source of concern. This patient's experience indicates the validity of that concern. However, one should also note that this elderly woman with severe disabling Parkinsonism of very long duration enjoyed a remarkable improvement which returned her, for nearly a year, to a useful independent existence.
CASE 2

F.H., a 62 year old man severely disabled by $\frac{\text { o }}{Z}$ advanced postencephalitic Parkinsonism, was ad-® mitted to the hospital in August 1969 for levodopa therapy. His history documented by U.S. Army and $\stackrel{\oplus}{=}$

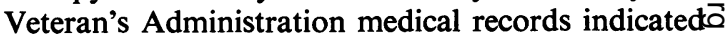
that he had suffered a febrile illness with somnolence ${ }_{0}^{D}$ in January 1944 treated at the time as an uppero respiratory infection. He recovered without apparent $\frac{\text {. }}{\text {. }}$. sequelae, returned to duty and subsequently received a routine discharge from the Army in September: 1945. Five years later he developed some tremor in $\overrightarrow{\overrightarrow{\vec{D}}}$ the left hand which later also involved the left lowero extremity. In 1953 he began to have oculogyrico crises in which his head would be retracted and $\overline{\bar{n}}$. turned to the left and his eyes deviated conjugately upwards and to the left. These symptoms gradually became more severe. The diagnosis of postencephal-s itic Parkinsonism was made and trihexyphenidyl $\overrightarrow{0}$ was prescribed. Gradual progression of this illness $\vec{a}$ with the development of bilateral tremor, rigidity, ${ }_{\sigma}^{\omega}$ slowness, impairment of gait and posture, ultimately compelled retirement from his work as a salesman:o By 1963, with further progression of the disease, the patient was severely invalided, being largely confinedp to bed and chair and requiring assistance in neaby all the activities of daily living. A left thalamotori $y$ was performed in 1964 with good relief of trempro and rigidity in the right upper extremity and sometransient improvement of gait and posture. How ever, he subsequently had a pronounced latega deviation of the trunk to the right of 10 to 15 degrees? and his illness continued to progress so that benefits of surgery had essentially disappeared within two years. Oculogyric crises continued to occur on the average of once per week on a regimen of trihexyphenidyl $20 \mathrm{mg}$ daily plus imipramine $100 \mathrm{mg}$ daily.

Laboratory investigations were remarkable only? for a very low concentration of cerebrospinal fluid homovanillic acid of $7 \mathrm{ng} / \mathrm{ml}$. Trihexyphenidyl was̄ continued and a trial of levodopa was begun at $\stackrel{3}{\not}$ dose of $0.5 \mathrm{~g}$ daily. The dosage was gradually increased to a daily total of $6 \mathrm{~g}$ over a period of one month. This dosage was well tolerated in hospitas and significant improvement was apparent; onlex two oculogyric crises had occurred during this period. Further improvement gradually accrued over. the following two months so that a very appreciable overall improvement was realized. The patien became independent in his daily living activities? he attended to his personal needs with minimal supervision, was able to perform minor chores and. was able to resume some social activities. Drooling completely ceased. Posture became almost normaP and speech was improved. For a brief period there 
was a sudden increase in the frequency of oculogyric crises which occurred almost daily and several times on some days. Because the increase was thought possibly to be due to levodopa; the dosage was reduced to $4 \mathrm{~g}$ daily. There was then some recrudescence of signs of Parkinsonism. The frequency of oculogyric crises declined and they remained infrequent thereafter, despite increases in the dosage of levodopa again to $6 \mathrm{~g}$ daily. However, after a month the dosage was again reduced because of the subsequent development of unusual behaviour which was very distressing to the patient's family. He exhibited numerous compulsions, the most striking of which was a frequent compulsion to polish and count the tiles on the bathroom wall. While shaving or brushing his teeth, the patient would seem to become fascinated with the tiles and would for long periods, sometimes an hour or more, continue to polish them with a wash cloth and count them. Similarly, while watching television he would become interested in the window drape and try endlessly to adjust the pleats. He became angry and resentful when his wife or son would try to stop these activities and on several occasions struck at them belligerently. He exhibited poor judgement, impulsiveness, and socially inappropriate behaviour. He promptly tried to sell to a neighbour a new sports jacket he had received as a gift. He tried to renew his automobile driver's licence and became abusive when his family objected. He telephoned old friends in the middle of the night to invite them to try to organize a party.

Various adjustments in the dosage were attempted but when the dosage was lowered below $5 \mathrm{~g}$ the resulting increase in severity of his Parkinsonism rendered him more of an invalid, while increases above $5 \cdot 25 \mathrm{~g}$ per day resulted in excessive compulsive and impulsive behaviour. The best compromise seemed to be about $5 \mathrm{~g}$ per day. Oculogyric crises remained markedly decreased throughout a $2 \frac{1}{2}$ year period in both severity and frequency. There were occasional transient tendencies to look up to the left but frank oculogyric crises were absent for periods lasting up to two to three months and they were quite mild, subsiding in 10 to 15 minutes.

COMMENT The compulsive behaviour exhibited by this patient is among the characteristic sequelae of encephalitis lethargica and was observed in a large proportion of its survivors. It was clearly increased by levodopa and consequently denied this patient the nearly complete reversal of the Parkinsonism that he might otherwise have enjoyed. Nevertheless, with careful adjustment of the dosage schedule of levodopa a compromise was found which produced some annoying behavioural aberrations but at the same time gave a very appreciable improvement of his Parkinsonism. A considerable period of time was required to achieve this satisfactory result.

The transient increase in oculogyric crises observed after three months of treatment in this patient was probably not related to levodopa, since oculogyria remained very mild and infrequent thereafter for a long follow up period of $2 \frac{1}{2}$ years.

\section{DISCUSSION}

Calne, Sharkey, and Armitage (1969) first reported the results of levodopa treatment in postencephalitic Parkinsonism. They administered maximum tolerated doses of levodopa to a group of 20 patients so disabled that institutional care had been necessary for many years. After six weeks of therapy, seven were substantially improved and three moderately so, while five patients had no useful response. The drug was discontinued in five patients because of sideeffects. The authors concluded that levodopa had a 'useful role' in the management of postencephalitic patients. However, in a subsequent communication dealing with the same patient population, Hunter, Stern, and Sharkey (1970) reported their impression that postencephalitic patients tolerate levodopa poorly, and stated that 'only a minority gain useful and enduring benefit'. Other authors (Krasner and Cornelius, 1970; Sacks et al., 1970a, b, c, and d) also found that postencephalitic patients had limited tolerance for levodopa. They drew attention particularly to the incidence of psychic disturbances, the occurrence of severe 'respiratory crises', and the reactivation of oculogyric crises.

Our experiences contradict these relatively negative assessments of levodopa. Over twothirds of our 30 patients obtained significant improvement over a prolonged period of observation and in most of these the benefits were very substantial. These results compare favourably with those obtained in Parkinson's disease. Several reasons may account for our more favourable experience. A longer period of treatment permitting careful individualization 
of dosage and dose-schedules is probably the major reason. Another may be that our population of patients differed with regard to the severity of their disease. Most of our patients attended an ambulatory outpatient clinic and only five were in stage IV or V of our disability scale.

In postencephalitic patients, as in those with Parkinson's disease, responsiveness to levodopa therapy does not seem related to age, or to the severity or duration of illness. However, it is our clinical impression that severely disabled patients may not profit as much as do mildly affected patients from a comparable degree of improvement in motor function. As in Parkinson's disease, adventitious involuntary movements and behavioural disturbances are the major doselimiting side-effects, frequently compelling a reduction of dosage with subsequent partial loss of the neurological improvement gained initially. We agree with the aforementioned authors that involuntary movements occur at a lower dosage of levodopa in postencephalitic Parkinsonism than in Parkinson's disease. At the same time, a favourable therapeutic response also occurs at a much lower dose than is required for patients with Parkinson's disease. The average daily dose of levodopa in our postencephalitic patients was $2.8 \mathrm{~g}$, whereas for Parkinson's disease patients the average is $5.5 \mathrm{~g}$ (Yahr, Duvoisin, Schear, Barrett, and Hoehn, 1969).

It is interesting to note that behavioural disorders are not always exacerbated and that demented patients may indeed derive significant benefit from levodopa. One must also note that behavioural disturbances occurring during levodopa therapy are not necessarily caused by this agent. Improvement in motor function can 'unmask' a psychosis or dementia not previously apparent. However, it is clear that in some patients the behavioural disorders typical of postencephalitic Parkinsonism are likely to be exacerbated by levodopa. It is interesting that in one of our patients abnormal behaviour which had been quiescent for many years was 'reactivated' by levodopa. A similar case has been described by Sacks et al. (1970a). We found in our patients a strict dose-dependency of psychic symptoms, and our successful experience with these patients suggests that careful dosage adjustment should be tried over a considerable period of time, in hospital if necessary, before one concludes that levodopa is useless in a given patient.

Although the oculogyric crises were not com pletely abolished except perhaps in one case, weo noted a marked reduction of frequency ande severity in six of nine patients and thus we cannots confirm the assertion of Sacks et al. (1970b) that severe reactivation of the oculogyria must be regarded as one of the most distressing side $-\overrightarrow{\vec{F}}$ effects of levodopa in these patients. The $\stackrel{?}{+}$ favourable effect of levodopa on oculogyria is? consistent with older reports of the efficacy of $\frac{\overline{0}}{\overline{0}}$ amphetamine in controlling this phenomenon in postencephalitic patients (Solomon, Mitchell, 임 and Prinzmetal, 1937). Many of the actions of amphetamine may be due to an enhancement of. dopaminergic neurones (Carlsson, 1970), giving $\vec{\omega}$ it a certain parallelism with the effects of levo- dopa which are ascribable to the dopamine to which it is transformed.

We did not observe any aggravation of the so- $\omega_{\pi}^{-}$ called 'respiratory crises', but in some patien abnormal respiratory patterns were seen as pa秙 of the choreiform syndrome induced by levodop?응 These were controlled by a reduction of the dose Our observations agree with those of Calne et At. (1969) that involuntary movements preseft before levodopa treatment are made worse.

Among the more striking minor symptoms postencephalitic Parkinsonism drooling stands $N$ out as one which may be markedly improved by응 levodopa, even in patients in whom large doses of anticholinergic drugs failed to yield a significant benefit. This observation confirms our impression in agreement with Parkinson (1817) that drooling in Parkinsonism is due to failure to $\overrightarrow{\overline{0}}$ direct the saliva posteriorly to the fauces-that is, drooling reflects a motor defect rather than sialorrhoea. The greater improvement of motor function yielded by levodopa than by previous anti-Parkinsonism remedies accounts for its? more remarkable effect on this distressing symptom.

Postural hypotension was encountered in only one patient. We may speculate that the involvement of the peripheral nervous system in Parkinson's disease, suggested by the presence of Lewy bodies in sympathetic ganglia (Jager and Bethlem, 1960), is not present in postencephalitic Parkinsonism, and that postencephalitic patients 
are consequently less prone to suffer changes in blood pressure regulation.

The very severe depletion of striatal dopamine found in a patient (case D), who came to necropsy, more severe than that found in our studies of patients with Parkinson's disease, is in agreement with the earlier observations of Hornykiewicz (1966). The very low concentration of homovanillic acid in the cerebrospinal fluid of our case 1 presumably reflects this severe dopamine depletion. These findings are consistent with the more severe degeneration of the substantia nigra seen in postencephalitic Parkinsonism (Greenfield and Bosanquet, 1953).

It is surprising, then, that postencephalitic patients respond to lower dosages of levodopa than do those with Parkinson's disease. One might have anticipated that these patients, having fewer surviving dopaminergic neurones, would have less opportunity to benefit from an augmented synthesis of dopamine. Yet their greater responsiveness to levodopa seems consistent with the observations of earlier clinical investigators working with amphetamine (Solomon et al., 1937) who noted beneficial results in postencephalitic but not in idiopathic Parkinsonism. This provocative difference in pharmacological response is difficult to explain. Presumably it reflects differences in the pathogenetic mechanism of dopaminergic neuronal dysfunction in the two forms of Parkinsonism. We may speculate that the virus of encephalitis lethargica damaged not only dopaminergic neurones but the receptor neurones as well, rendering them hypersensitive to dopamine. However, we should remain cautious about ascribing all the manifestations of Parkinson's disease to striatal dopamine depletion alone.

\section{REFERENCES}

Calne, D. B., Stern, G. M., Laurence, D. R., Sharkey, J., and Armitage, P. (1969). L-dopa in postencephalitic Parkinsonism. Lancet, 1, 744-747.
Carlsson, A. (1970). Amphetamine and brain catecholamines. In International Symposium on Amphetamines and Related Compounds, Milan, pp. 289-300. Edited by E. Costa and S. Garattini. Raven Press: New York.

Cote, L., Yahr, M. D., Duvoisin, R. C., Wolf, A., and Marksbery, W. (1970). Analysis of deaths in Parkinson patients on long-term levodopa therapy. Transactions of the American Neurological Association, 95, 73-77

Duvoisin, R. C., and Yahr, M. D. (1965). Encephalitis and Parkinsonism. Archives of Neurology, 12, 227-239.

Greenfield, J. G., and Bosanquet, F. D. (1953). The brainstem lesions in Parkinsonism. Journal of Neurology, Neurosurgery, and Psychiatry, 16, 213-226.

Hoehn, M. M., and Yahr, M. D. (1967). Parkinsonism: onset, progression, and mortality. Neurology (Minneap.), 17, 427-442.

Hornykiewicz, O. (1966). Metabolism of brain dopamine in human Parkinsonism: neurochemical and clinical aspects. In Biochemistry and Pharmacology of the Basal Ganglia, pp. 171-185. Edited by E. Costa, L. J. Côté, and M. D. Yahr. Raven Press: New York.

Hunter, K. R., Stern, G. M., and Sharkey, J. (1970). Levodopa in postencephalitic Parkinsonism. Lancet, 2, 13661367.

Jager, W. A. den H., and Bethlem, J. (1960). The distribution of Lewy bodies in the central and autonomic nervous systems in idiopathic paralysis agitans. Journal of Neurology, Neurosurgery, and Psychiatry, 23, 283-290.

Krasner, N., and Cornelius, J. M. (1970). L-dopa for postencephalitic Parkinsonism. British Medical Journal, 4, 496.

Parkinson, J. (1817). An Essay on the Shaking Palsy, p. \&. Sherwood, Neely, and Jones: London.

Sacks, O. W., and Kohl, M. (1970a). Incontinent nostalgia induced by L-dopa. Lancet, 1, 1394.

Sacks, O. W., and Kohl, M. (1970b). L-dopa and oculogyric crises. Lancet, 2, 215-216.

Sacks, O. W., Kohl, M., Schwartz, W., and Messeloff, C. (1970c). Side-effects of L-dopa in postencephalitic Parkinsonism. Lancet, 1, 1006.

Sacks, O. W., Messeloff, C. R., and Schwartz, W. F. (1970d). Long-term levodopa in the severely disabled. Journal of the American Medical Association, 213, 2270.

Solomon, P., Mitchell, R. S., and Prinzmetal, M. (1937). The use of benzedrine sulfate in postencephalitic Parkinson's disease. Journal of the American Medical Association, 108, 1765-1770.

Yahr, M. D., Duvoisin, R. C., Schear, M. J., Barrett, R. E., and Hoehn, M. M. (1969). Treatment of Parkinsonism with levodopa. Archives of Neurology (Chicago), 21, 343354. 